\title{
Capacidade de carga na trilha principal da ARIE da Floresta da Cicuta, Volta Redonda-Barra Mansa, Rio de Janeiro, Brasil
}

Tobias Lee, Lundoi ${ }^{1,4}$; Luciana Cristina do Carmo Silva Carvalho ${ }^{2}$; Camila Riquete Coelho'; Lucinere de Souza Quintanilha Carvalho²; Sabrinna Aires Garcia²; Márcia Valéria da Fonseca Porto ${ }^{3}$; Sandro Leonardo Alves ${ }^{3}$; Welington Kiffer de Freitas ${ }^{2}$

${ }^{1}$ Departamento de Microbiologia Agrícola, Universidade Federal de Lavras, Minas Gerais, Brasil; ${ }^{2}$ Escola de Engenharia Industrial Metalúrgica de Volta Redonda, Universidade Federal Fluminense, Volta Redonda, Rio de Janeiro, Brasil; ${ }^{3}$ Instituto Chico Mendes de Conservação da Biodiversidade (ICMBio), Volta Redonda, Río de Janeiro, Brasil; ${ }^{4}$ lundoilee@id.uff.br

Tobias Lee, Lundoi; Luciana Cristina do Carmo Silva Carvalho; Camila Riquete Coelho; Lucinere de Souza Quintanilha Carvalho; Sabrinna Aires Garcia; Márcia Valéria da Fonseca Porto; Sandro Leonardo Alves; Welington Kiffer de Freitas (2019) Capacidade de carga na trilha principal da ARIE da Floresta da Cicuta, Volta Redonda-Barra Mansa, Rio de Janeiro, Brasil. Rev. Fac. Agron. Vol 118 (2): 1-10. https://doi.org/10.24215/16699513e021

O presente estudo foi realizado na ARIE da Floresta da Cicuta, Barra Mansa e Volta Redonda (RJ). Teve como objetivo determinar e avaliar o potencial de gestão e a Capacidade de Carga Turística da Trilha Principal da ARIE dessa UC, gerando um valor estimado do número de visitantes por dia, complementando o Plano de Manejo da referida ARIE. Para a determinação de capacidade de carga turística da trilha principal da ARIE foi aplicado o método proposto por Cifuentes (1992). O presente estudo demonstrou que a trilha está, relativamente, bem alinhada com o SNUC. A Capacidade de Carga Efetiva encontrada para a trilha foi de 208 visitas $x$ dia ${ }^{-1}$. Esse estudo poderá contribuir com o processo de atualização do Plano de Manejo da ARIE, sendo complementado com outros estudos.

Palavras-chave: uso público, impacto ambiental, manejo, conservação, recreação ao ar livre

Tobias Lee, Lundoi; Luciana Cristina do Carmo Silva Carvalho; Camila Riquete Coelho; Lucinere de Souza Quintanilha Carvalho; Sabrinna Aires Garcia; Márcia Valéria da Fonseca Porto; Sandro Leonardo Alves; Welington Kiffer de Freitas (2019) Determination on the carrying capacity in the Main Trail of Cicuta's ARIE, Volta Redonda-Barra Mansa, Rio de Janeiro, Brasil. Rev. Fac. Agron. Vol 118 (2): 1-10. https://doi.org/10.24215/16699513e021

The present study was carried out in the ARIE of the Cicuta Forest, Barra Mansa and Volta Redonda (RJ). The objective was to determine the tourism tarrying capacity of the Main Trail of the ARIE, generating an estimated number of visitors per day, and to evaluate the management potential to complement the Management Plan. The method proposed by Cifuentes (1992) was applied to determine the tourist carrying capacity. The present study demonstrated that the trail is relatively well aligned with the National System of Protected Areas (SNUC). The Effective Carrying Capacity was 208 visitors $x_{\text {day }}^{-1}$. This study may contribute to the process of updating the ARIE Management Plan, but should be complemented with other studies (Acceptable Exchange Limit - AEL, Visitor Impact Management - VIM, etc.).

Keywords: public use, environmental impact, management, conservation, outdoor recreation

https://revistas.unlp.edu.ar/revagro

Recibido: 19/07/2018

Aceptado: $16 / 08 / 2019$

Disponible on line: $27 / 12 / 2019$

ISSN 0041-8676 - ISSN (on line) 1669-9513, Facultad de Ciencias Agrarias y Forestales, UNLP, Argentina 


\section{INTRODUÇÃO}

O Sistema Nacional de Unidades de Conservação (SNUC), regulamentado pela Lei Federal $\mathrm{N}^{\circ}$ 9.985/2000, define Unidade de Conservação (UC) como "espaço territorial e seus recursos ambientais, incluindo as águas jurisdicionais, com características naturais relevantes, legalmente instituído pelo Poder Público, com objetivos de conservação e limites definidos, sob regime especial de administração, ao qual se aplicam garantias adequadas de proteção" (Brasil, 2000).

Considerando apenas as UCs Federais, sob tutela do Instituto Chico Mendes de Conservação da Biodiversidade (ICMBio), o território nacional encontrase protegido por mais de 1 milhão de $\mathrm{Km}^{2}$ com diferentes categorias de manejo e, somente o ano de 2017, registrou a visita de cerca de 10 milhões de pessoas (ICMBio, 2018).

Dentre as categorias de manejo previstas pelo SNUC, encontram-se as Áreas de Relevante Interesse Ecológico (ARIEs), pertencente ao grupo de Uso Sustentável. As ARIEs podem ser implementadas em terras públicas ou privadas. Em geral, possuem pequena extensão, com pouca ou nenhuma ocupação humana, com características naturais extraordinárias ou que abriguem exemplares raros da biota regional, objetivando manter os ecossistemas naturais, de importância, regional ou local, buscando compatibilizar os objetivos de conservação com atividades desenvolvidas pela sociedade (Brasil, 2000). De acordo com o SNUC, nessas áreas, respeitados os limites legais, podem ser estabelecidas normas e restrições para a utilização de uma propriedade, podendo ser permitidas a realização de pesquisa científica e a visitação, de acordo com a regulamentação estabelecida pelo Plano de Manejo (Brasil, 2000).

O Plano de Manejo é um documento técnico baseado em atributos biogeofísicos e socioeconômicos, o qual estabelece o zoneamento da UC, conforme os objetivos de manejo, para servir como referência de manejo e gestão das UCs, inclusive para o direcionamento da implantação de estruturas físicas, por exemplo, sistema de trilhas (Brasil, 2000). Conforme Bonatti et al. (2006); Souza \& Martos (2008), quando os Planos de Manejo não atendem efetivamente a vocação para o uso público podem inviabilizar o potencial de visitação e recreação das UCs pertencentes a categorias de manejo que preveem essas atividades.

As atividades de uso público em UCs e outras áreas naturais protegidas podem resultar em importantes serviços ecossistêmicos para a nossa sociedade, mas essas atividades também podem induzir efeitos indesejáveis em vários componentes ecológicos, além de comprometer a experiência dos visitantes (Marion et al., 2016).

Nesse aspecto, a capacidade de carga turística (CCT) é uma ferramenta de gestão ambiental que visa determinar o número de visitantes em um determinado local, por um período de tempo, onde se realizam estimativas baseadas em parâmetros específicos, com o objetivo de mitigar as externalidades negativas provenientes das atividades de uso público sobre o ambiente natural, sem prejuízo na qualidade e na experiência dos visitantes (Gil et al., 2014; Soria-Díaz,
2013). A expressão capacidade de carga foi adaptada para o manejo das atividades de uso público em áreas naturais a partir de estudos realizados pelos pesquisadores de produção animal para definir a quantidade de animais que uma determinada área, expressa em ha, pode sustentar como fonte de recurso para a pastagem (Dal Soller \& Borghetti, 2013).

Inicialmente, Cifuentes (1992) propôs uma metodologia para a determinação da Capacidade Carga em áreas naturais. Em seu estudo, na Reserva Biológica de Carrara (Costa Rica), foi estimada a Capacidade de Carga Efetiva (CCE) para o uso público na trilha "Quebrada Bonita", considerando fatores de correção (FC) como: duração do dia, precipitação, erodibilidade do solo, acessibilidade dos usuários, distúrbios da fauna e temporariedade, chegando ao número de 11 visitantes $\mathrm{x} \mathrm{dia}^{-1}$.

Desde então, esse método foi sendo disseminado para o mundo, sendo aplicado em diferentes áreas envolvidas com o uso público. Amador et al. (1996) analisaram diferentes sítios de visitação no Parque Nacional de Galápagos (Equador); Sayan \& Atik (2011) avaliaram rede de trilhas do Parque Nacional de Termessos (Turquia); Ríos-Jara et al. (2013) aplicaram o método nas trilhas sub aquáticas no Parque Nacional da Ilha Isabel (México); Queiroz et al. (2014) nas trilhas de pedestres do sítios da rede Natura 2000 nas ilhas do Açores (Portugal); Cimnaghia \& Mussini (2015) aplicaram o método para investigar o fluxo turístico e uso dos espaços da Galeria Nacional de Marche e do museu de artes do século XXI (Itália); Ibanez Pérez (2016) estimou a carga em unidades de manejo ambiental para o ecoturismo em Baja California Sur (México), dentre outros. No Brasil, muitos estudos também se apoiaram no método descrito por Cifuentes para o manejo da visitação em áreas protegidas: Bonatti et al. (2006), nas trilhas da Floresta Nacional São Francisco de Paula (RS); Ruschmann et al. (2008), no planejamento turístico Praia Brava (Itajaí, SC) e outros.

Diante disso, o presente trabalho objetivou determinar e avaliar o potencial de gestão e a Capacidade de Carga Turística da trilha principal da ARIE da Floresta da Cicuta, localizada nos municípios de Volta Redonda e Barra Mansa, RJ, gerando um valor estimado do número de visitantes por dia, contributo para a gestão sustentável da área, e destarte, complementando o Plano de Manejo da referida UC.

\section{MATERIAL E MÉTODOS}

\section{Área de estudo}

A ARIE da Floresta da Cicuta possui 131 ha e está inserida na sub-bacia do Médio Paraíba, Brasil, entre as coordenadas $22^{\circ} 32^{\prime \prime} 28.08^{\prime \prime}$ e $22^{\circ} 33^{\prime \prime} 27.32$ "S e $44^{\circ} 05^{\prime \prime} 42.74^{\prime \prime}$ e $44^{\circ} 05^{\prime \prime} 0.66^{\prime \prime} \mathrm{O}$, nos limites dos municípios de Barra Mansa e Volta Redonda (Souza et al., 2007).

O histórico de criação da ARIE da Floresta da Cicuta remonta a década de 1940. Nessa fase, a Fazenda Santa Cecília, integrante do patrimônio da Companhia Siderúrgica Nacional (CSN), manteve-se aberta à visitação pública até o ano de 1972. A partir dessa data, a área foi fechada pela própria $\mathrm{CSN}$, objetivando a 
preservação ambiental. A ARIE da Floresta da Cicuta foi criada, de fato, através do Decreto Federal $\mathrm{N}^{\circ}$ 90.972/85. A partir disso a gestão passou para o Instituto Brasileiro do Meio Ambiente e dos Recursos Naturais Renováveis (IBAMA), visando a proteção de um dos últimos remanescentes da Floresta Estacional Semidecidual na região do médio Paraíba do Sul, Rio de Janeiro. Em 1993, a CSN foi privatizada e, como o perímetro legal da UC não foi ressalvado, passou a constituir um modelo misto de gestão, envolvendo a proprietária da terra (CSN) e o órgão ambiental (IBAMA). Posteriormente, em 2007, a ARIE passou para a tutela do Instituto Chico Mendes de Conservação da Biodiversidade (ICMBio, 2016).

A vegetação da região é caracterizada como Floresta Estacional Semidecidual Submontana. Na ARIE predominam os mesofanerófitos, com a participação de megafanerófitos sobressaindo na linha do dossel arbóreo, com destaque para as espécies pertencentes à família Euphorbiaceae - Actinostemon klotzschii (Didr.) Pax, Senefeldera verticillata (Vell.) Croizat e Maprounea guianensis Aubl. (Souza et al., 2007).

O relevo da região caracteriza-se como "mar de morro", pertencentes às unidades geomorfológicas de planície de leito fluvial, formada por sedimentos dispostos no fundo do vale, além de frequentes afloramentos cristalinos, provavelmente, formado por rochas granítico-gnáissicas. A concentração de pequenos vales proporcionou o assentamento urbano e industrial das cidades de Volta Redonda e Barra Mansa (ICMBio, 2016).

O clima predominante é o mesotérmico (Cwa), inverno seco e verão quente e chuvoso, com elevados índices de umidade $(77 \%)$, mesmo nos meses de inverno (Souza et al., 2007). Conforme Fonseca (2018), nessa região, os meses de novembro a março compreende o período mais chuvoso e mais quente no município.

A trilha principal é o mobiliário mais utilizado no programa de uso público na ARIE da Floresta da Cicuta. Essa trilha possui um traçado linear, com 1.753 $\mathrm{m}$ de extensão, os quais os visitantes percorrem em 3 horas, considerando o tempo de ida e volta, incluindo atividades de educação e interpretação ambiental, desenvolvidas pela equipe da ARIE. O percurso compreende desde a entrada, na placa de criação da ARIE até a cachoeira do rio Brandão, afluente do Paraíba do Sul. O critério arbitrado durante a oficina de planejamento (OOP) do Plano de Manejo, em 2006, após a avaliação dos pesquisadores e representantes do ICMBio envolvidos no processo, definiu que as atividades na trilha seriam de dois grupos por dia, com 20 visitantes cada grupo (Figura 1). A trilha apresenta baixo grau de dificuldade, recomendada para todas as idades, sendo inapropriada para o trânsito de cadeirantes, devido à irregularidade do terreno e à falta de infraestrutura adequada para visitantes com deficiência física.

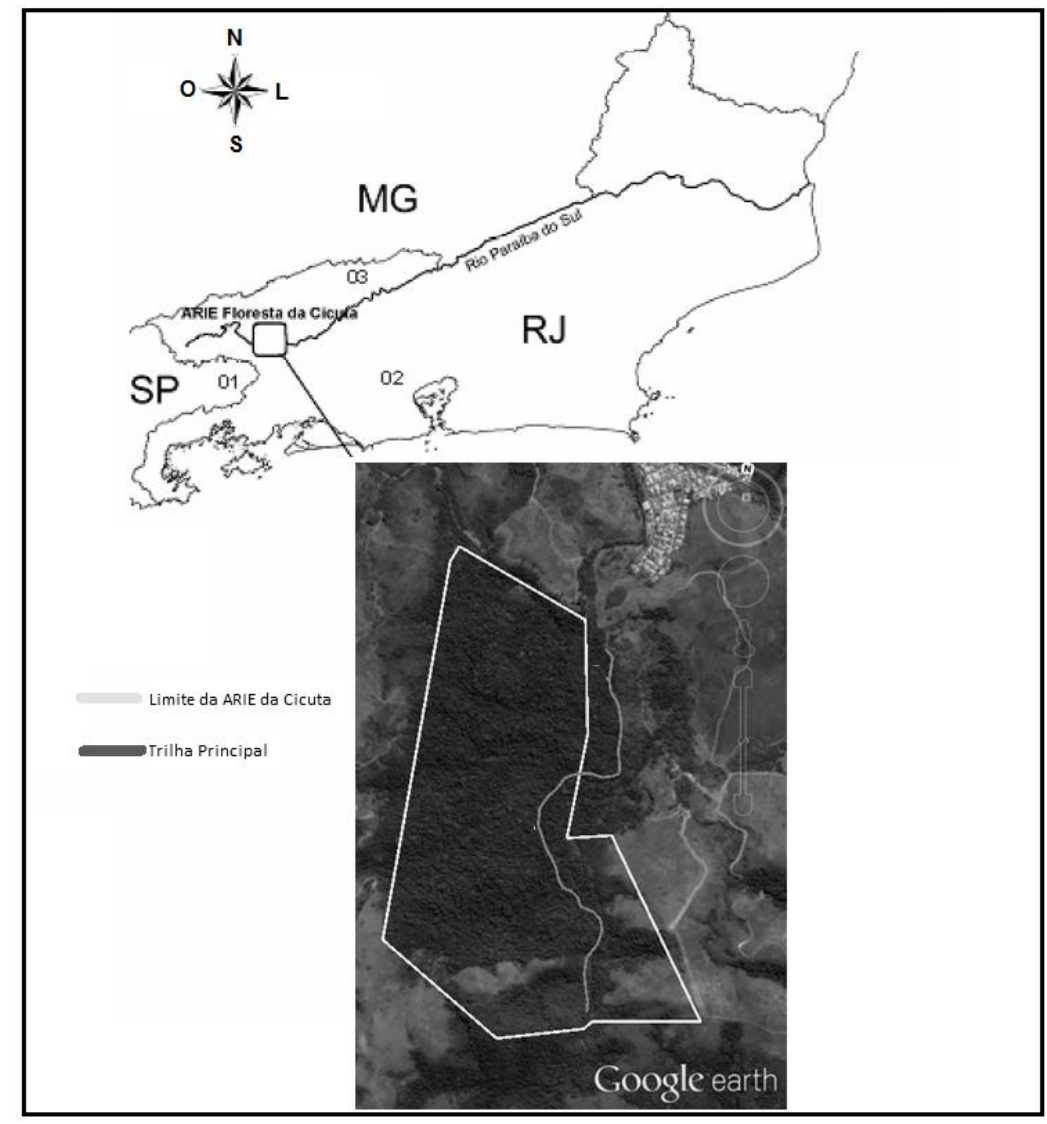

Figura 1. Localização da ARIE e da Trilha Principal da Floresta da Cicuta, Volta Redonda, Barra Mansa, RJ. Adaptado de Souza et al. (2007). 


\section{Aspectos metodológicos}

Para a realização da avaliação doas aspectos da gestão da UC foram considerados documentos oficiais (Plano de Manejo da UC, relatórios das Oficinas de Planejamento Participativo - OPP e site do ICMBio), Roteiros Metodológicos (RAPPAN), artigos publicados em periódicos, além de consulta a equipe gestora.

A determinação da Capacidade de Carga da Trilha Principal da ARIE da Floresta da cicuta foi realizada durante o mês de maio de 2016. Todo o traçado da trilha foi subdivido em 24 seções, com 73 metros cada, demarcadas com bandeirolas afixadas no solo, confeccionadas com arame e plástico vermelho. Ao longo de cada seção, foram observadas características, como: largura da trilha, declividade, alagamento, presença de atalhos, sinais de erosão do solo, tipo de piso, interferências antrópicas (depósito de resíduos, inscrições em árvores, infraestrutura, características da vegetação e espécies notáveis da flora). Para a coleta dos dados foram utilizados: trena de $50 \mathrm{~m}$, altímetro digital, GPS Garmin Etrex 2.2, clinômetro do tipo Suunto. Todos os registros foram feitos em caderneta de campo. Depois de concluído o trabalho de campo, procedeu-se a fase de pesquisa documental, durante os meses de junho a outubro de 2016, através da consulta de relatórios técnicos fornecidos pela administração da UC, diplomas legais e artigos científicos. A pesquisa foi desenvolvida de acordo com a metodologia proposta por Cifuentes (1992), sintetizada na Figura 2.

Para a determinação de capacidade de carga turística, metodologia na qual o presente estudo se baseou, têmse:

Capacidade de Carga Física (CCF): é o limite máximo de visitas que se pode fazer em um local com espaço definido, em um tempo determinado. Podendo ser expressa com a fórmula geral:

Onde:

$$
\mathrm{CCF}=\left(\mathrm{V} \times \mathrm{A}^{-1}\right) \times \mathrm{s} \times \mathrm{t} \quad(\mathrm{Eq} .1)
$$

$\mathrm{V} \times \mathrm{A}^{-1}$ : Visitantes por área ocupada.

s: Superfície disponível para uso público.

t: Tempo necessário para efetuar a visita.

\section{Premissas}

1. Segundo Cifuentes (1992) estima-se que uma pessoa requer $1 \mathrm{~m}^{2}$ de espaço para se mover livremente;

2. O tempo necessário para a visitação na trilha tem duração de 3 horas, incluindo as atividades de interpretação guiada e contemplação da cachoeira (ICMBio, 2016).

Capacidade de Carga Real (CCR): é o limite máximo de visitas, determinado a partir da CCF de um lugar, após submetê-lo aos fatores de correção definidos em função das características particulares do lugar. Os fatores de correção são obtidos considerando variáveis físicas, ambientais, ecológicas, sociais e de manejo. Podendo ser expressa pela fórmula geral:

Figura 2. Processo de Planejamento para manejo de Visitação em Unidades de Conservação. Adaptado de Cifuentes (1992).

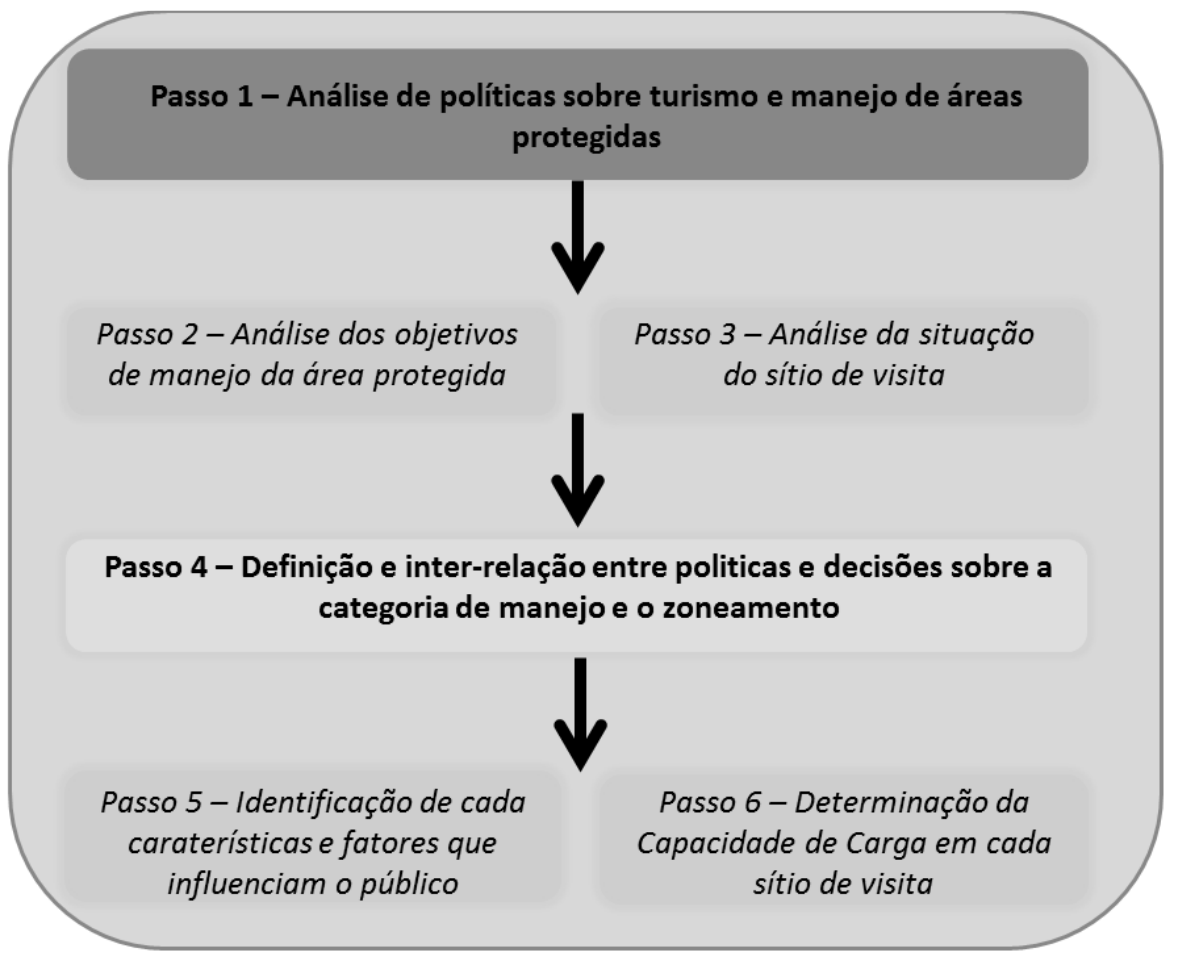


Revista de la Facultad de Agronomía, La Plata (2019) Vol 118 (2): 1-10

$$
\mathrm{CCR}=(\mathrm{CCF} \times \mathrm{FC}) \quad(\text { Eq. } 2)
$$

Fatores de Correção (FC):

a) $\quad F C_{B S}$ - Brilho Solar. A radiação no período de um dia é bem mais intensa entre $12 \mathrm{hs}$ às $14 \mathrm{hs}$, o que dificulta a visitação, por exemplo, em sítios sem cobertura do dossel florestal (Cifuentes, 1992).

Onde:

$\mathrm{Mi}=$ Radiação no horário da visita

$\mathrm{Mt}=$ Radiação total na ARIE

$$
\mathrm{FC}_{\mathrm{BS}}=1-\mathrm{Mi} \times \mathrm{Mt}^{-1}=(\%) \quad \text { (Eq. 2.1) }
$$

MI - Magnitude limitante / Mt - Magnitude total

Premissas

1. Hora de brilho solar durante a visitação

b) $\quad F C_{E}-$ Erodibilidade: Esse fator busca analisar o efeito da erosão potencial e efetiva no sítio de visitação. Uma trilha se torna mais ou menos suscetível à erosão de acordo com o tipo de solo (TS), a declividade (D) do terreno e o tipo de atividade que nela se desenvolve. A aplicação dos fatores de correção para erodibilidade é um critério de decisão de manejo da equipe responsável, sendo recomendado o fator 2 para os trechos com média vulnerabilidade à erosão e 3 para os trechos críticos (Cifuentes, 1992; Mitraud, 2003).

Onde:

$\mathrm{Mi}=\sum_{i=1}^{i=n}(a t+b i)=(\mathrm{m})$

$a_{i}=$ trechos com fator $2 / b_{i}=$ trechos com fator 3

$\mathrm{Mt}=$ Comprimento total da trilha $(\mathrm{m})$

$$
\mathrm{FC}_{\mathrm{E}}=1-\left(\mathrm{Mi} \times \mathrm{Mt}^{-1}\right)=(\%) \quad(\mathrm{Eq} .2 .2)
$$

Ml - Magnitude limitante / Mt - Magnitude total

Premissas

$\begin{array}{lcl}\text { TS D } \leq 10 \% & 10 \%<\mathrm{D} \leq 20 \% & \mathrm{D}>20 \% \\ \text { Pedregoso Baixa } & \text { Média } & \text { Alta } \\ \text { Argiloso Baixa } & \text { Média } & \text { Alta } \\ \text { Areno-argiloso Baixa } & \text { Média } & \text { Alta }\end{array}$

c) $\quad F C_{A}$ - Acessibilidade: Refere-se ao grau de dificuldade que os visitantes poderiam encontrar em seus deslocamentos durante o percurso da trilha (Cifuentes, 1992).

Onde:

$\mathrm{Mi}=\sum_{i=1}^{i=n}(a t+b i+c i)=(\mathrm{m})$

$a_{i}=$ fator $1 / b_{i}=$ fator $1,5 / \mathrm{ci}=$ fator 2

$\mathrm{Mt}=$ Comprimento total da trilha $(\mathrm{m})$

$$
\mathrm{FC}_{\mathrm{A}}=1-\left(\mathrm{Mi} \times \mathrm{Mt}^{-1}\right)=(\%) \quad(\text { Eq. } 2.3)
$$

MI - Magnitude limitante / Mt - Magnitude total

Premissas

$$
\begin{aligned}
& D \leq 10 \% \text { - Fator } 1 \\
& 10 \%<D \leq 20 \%-\text { Fator } 1,5 \\
& D>20 \% \text { - Fator } 2
\end{aligned}
$$

d) $\quad F C_{P}$ - Precipitação: Com base no climograma abaixo (Figura 3) foram estabelecidos os dias em que a trilha deve permanecer em funcionamento e os dias em que a visitação deve ser interrompida, em épocas que as séries históricas apresentam maiores riscos de chuvas fortes, especialmente, no período vespertino na região.

Onde:

$\mathrm{Mi}=$ Trilha fechada (dia)

Mt = Trilha aberta (dia)

$$
\mathrm{FC}_{\mathrm{p}}=1-\left(\mathrm{Mi} \times \mathrm{Mt}^{-1}\right)=(\%) \quad(\text { Eq. } 2.4)
$$

MI - Magnitude limitante / Mt - Magnitude total

Premissas

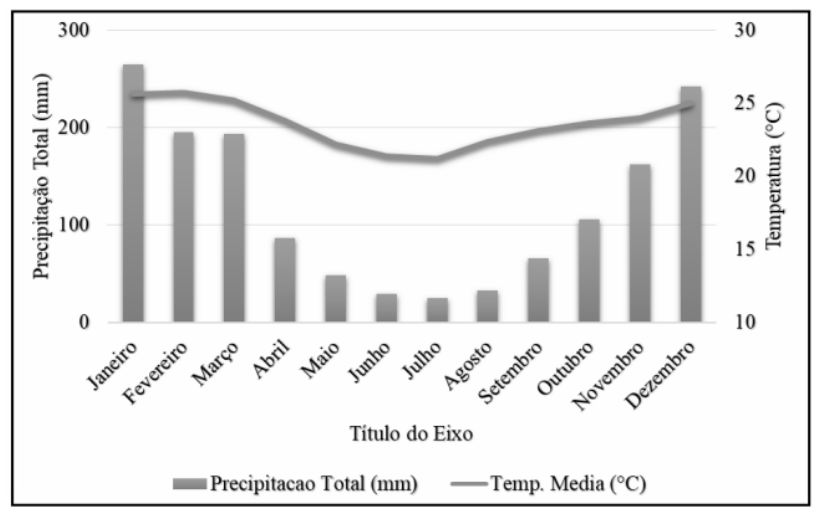

Figura 3. Climograma para a série histórica de temperatura e precipitação em Volta Redonda-RJ (1967 - 2016). Fonte: Fonseca (2018).

e) $\quad F C_{F}$ - Fauna: Na ARIE da Floresta da Cicuta podem ser encontrados exemplares da fauna como o bugio (Alouatta clamitans) e a jaguatirica (Felis pardalis ou Leopardus pardalis), ambos ameaçados de extinção na região (ICMBio, 2016). Portanto, deve-se evitar a circulação de pessoas, uma vez que que qualquer distúrbio no ambiente pode interferir no processo de reprodução dessas espécies (Cifuentes, 1992).

Onde:

Mi = Período de reprodução (mês)

Mt $=$ Ciclo de avaliação (mês)

$$
\mathrm{FC}_{\mathrm{F}}=1-\left(\mathrm{Mi} \times \mathrm{Mt}^{-1}\right)=(\%) \quad(\mathrm{Eq} .2 .5)
$$

Ml - Magnitude limitante / Mt - Magnitude total 
f) $\quad F C_{S T}-$ Series Temporais: Segundo Cifuentes (1992) esse fator representa o tempo necessário para as atividades de manutenção do sítio em questão, em relação à drenagem, controle de erosão, pontes, travessias, fechamento de desvios, sinalização, desobstrução de traçado, etc.

Onde:

$\mathrm{Mi}=$ Período de manutenção da trilha (dia)

$\mathrm{Mt}=$ Período de visitação (dia)

$$
\mathrm{FC}_{\mathrm{ST}}=1-\left(\mathrm{Mi} \times \mathrm{Mt}^{-1}\right)=(\%) \quad(\text { Eq. } 2.6)
$$

Ml - Magnitude limitante / Mt - Magnitude total

O conceito de CCR permite estimar as experiências dos visitantes considerando implicações físicas, ecológicas e sociais (Cupul-Magaña \& Rodríguez-Troncoso, 2017). No entanto, a carência de infraestrutura física e a capacidade de gestão das áreas protegidas vem comprometendo a concomitância das atividades de uso público com o cumprimento dos objetivos de conservação dessas áreas (Canto-Silva \& Silva, 2017). Por isso, Cifuentes (1992) recomenda que seja levada em conta a capacidade de manejo mínima indispensável para se estabelecer um percentual referente à Capacidade de Carga Real, considerando variáveis como respaldo jurídico e político, equipamentos disponíveis, dotação de pessoal, orçamento anual e condições de infraestrutura para a manutenção e atendimento ao visitante. Segundo Bonatti et al. (2006), a Capacidade de Manejo (CM), é um fator expresso em porcentagem, que está relacionado com variáveis tais como: recursos humanos, orçamento anual, condições de infraestrutura e outras.

$$
\mathrm{CCE}=\mathrm{CCR} \times \mathrm{CM}(\mathrm{Eq} .2 .7)
$$

\section{RESULTADOS E DISCUSSÃO}

A Convenção sobre a Diversidade Biológica (CDB) exige que as áreas protegidas sejam "gerenciadas de forma efetiva e equitativa". No entanto, a implementação de iniciativas de conservação não é a garantia de sucesso ecológico ou de benefício para os seres humanos (Bennett, 2016). Conforme Medeiros \& Pereira (2011) a gestão dessas áreas deve ser realizada a partir de um processo contínuo e necessário para que efetivamente se cumpram os objetivos estabelecidos para o seu manejo.

A análise de políticas sobre as Unidades de Conservação demonstra que o objetivo principal da categoria de manejo ARIE é a conservação de recursos naturais de relevância regional, normalmente em áreas com baixa ocupação humana (Brasil, 2000). Conforme Nascimento et al. (2016), nas categorias de manejo em que o uso público está previsto, como as ARIEs, os gestores devem também proporcionar aos visitantes a oportunidade de conhecer, entender e valorizar os recursos naturais e culturais existentes nesses espaços.
As diretrizes para visitação em Unidades de Conservação, estabelecidas pelo Ministério do Meio Ambiente (MMA, 2006), ressaltam que as atividades de uso público devem estar claramente regulamentadas pelo Plano de Manejo, considerando: o perfil do público; o zoneamento da área; busca de infraestrutura e equipamentos adequados, incluindo técnicas de monitoramento dos indicadores vinculados aos impactos provenientes dessas atividades aliado à satisfação dos visitantes.

De acordo com o resultado do estudo intitulado "Avaliação Rápida e a Priorização do Manejo de Unidades de Conservação das UCs Federais" (Rapid Assessment and Prioritization of Protected Area Management - RAPPAM), a ARIE da Floresta da Cicuta encontra-se relativamente bem alinhada, atingindo um índice de efetividade de manejo de $41 \%$. Esse estudo aponta os maiores destaques para a importância biológica da área $(89 \%)$, comunicação e informação (83\%), infraestrutura $(76 \%)$ e cumprimento dos objetivos de manejo (67\%). Por outro lado, os aspectos relacionados ao planejamento, gestão, pesquisa, avaliação e monitoramento foram normativas gerais tratadas pelo SNUC, enquanto fatores que não pontuaram na ocasião do estudo (ICMBio, 2011). No entanto, hoje, após o empenho da equipe gestora da Floresta da Cicuta no sentido de aprovar o Plano de Manejo, instituir Conselho Gestor, estabelecer um programa de visitação guiada continuada para grupos específicos, como: escolas, grupos religiosos, agentes comunitários, etc (ICMBio, 2016), a efetividade de manejo da UC atingiu pontuações representativas nos quesitos mal classificados naquela ocasião.

A ampliação e a atualização desses quesitos de manejo devem contar com constantes investimentos, inclusive aqueles relacionados à visitação. Segundo Medeiros \& Pereira (2011), a visitação pública em UCs pode gerar uma série de benefícios, porém, quando mal planejada e gerida, também causar riscos e impactos.

Watson et al. (2014) afirmam que a falta de investimentos, seja de recursos humanos ou financeiros, afetam 0 desempenho das áreas protegidas, inclusive interferindo no processo de demarcação de fronteiras, aplicação efetiva da lei, manejo de recursos naturais e culturais e a provisão de infraestrutura adequada para o manejo das UCs, o que em conjunto pode tornar essas áreas desinteressantes para a sociedade. Um fato de extrema importância para a consolidação de um programa de uso público em UCs refere-se aos recursos humanos, com capacitação continuada da equipe sobre temas correlacionados com o uso público (Bernard et al., 2014; MMA, 2006). Marion et al. (2016) ressaltam que o investimento nas áreas protegidas se justifica, pois a gestão profissional dos impactos dos visitantes requer um conhecimento profundo sobre os diferentes tipos de impactos, sua gravidade, extensão, distribuição espacial, e a influência de fatores, alguns dos quais são fatores causais, como a quantidade de uso e o comportamento visitante, e outros são fatores não-causais, tais como suscetibilidade ambiental.

O zoneamento da ARIE da floresta da Cicuta, proposta pelo Plano de Manejo, define três zonas. A Zona de Amortecimento que compreende o entorno da ARIE, onde as atividades humanas estão sujeitas às normas $\mathrm{e}$ 
restrições específicas, com o propósito de minimizar os impactos negativos sobre a UC. Uma Zona de Proteção, que compreende $77 \%$ da ARIE, contendo áreas naturais ou que tenham recebido grau mínimo de intervenção humana. $E$, finalmente, a Zona de Recuperação que apresenta em seus limites áreas antropizadas: linha de transmissão e infestações de espécies exóticas (capim colonião, bambus), margeando o rio Brandão, compreendendo $18 \%$ da UC. Essa classificação possui caráter transitório, já que uma vez recuperada será incorporada novamente à Zona de Proteção. Ríos-Jara et al. (2013), Malik \& Bhat (2015) afirmam que em ecossistemas frágeis devido ao tamanho, como no caso da trilha principal da ARIE da Cicuta, é importante estabelecer um zoneamento apropriado, com critérios para o uso dos serviços ecossistêmicos de acordo com suas potencialidades, considerando suas implicações socioambientais.

O traçado da trilha principal projeta-se justamente na área de contato entre as Zonas de Proteção e de Recuperação, com início no marco de criação da ARIE até a cachoeira do rio Brandão. Em seu percurso os visitantes apreciam aspectos cênicos que a UC oferece em termos de fauna e flora. Entre as espécies da flora foram encontrados vários exemplares de grande importância, como o jequitibá-rosa (Cariniana legalis), jatobá (Hymenaea courbaril), além de uma figueira centenária (Ficus cyclophylla). No fim dessa trilha, está localizado o principal ponto de interesse da visitação, a cachoeira do Rio Brandão.

Nesse estudo os fatores sociais não foram considerados nos cálculos, porque a equipe da ARIE já adota como procedimento para visitação nessa trilha a formação de grupos com, no máximo, 10 indivíduos, partindo da premissa que cada indivíduo necessita de $1 \mathrm{~m}^{2}$ de trilha para seu conforto e segurança e que a distância mínima entre dois grupos não seja inferior a $100 \mathrm{~m}$. Ademais, as atividades relacionadas ao monitoramento e à manutenção podem ser realizadas pela própria equipe da ARIE e grupo de voluntários da UC, já que os grupos só visitam a trilha três vezes por semana. O que pressupõe que não há danos ou deterioração causados pelos visitantes durante o visita. Com isso, a determinação da Capacidade de Carga Turística para a trilha Principal foi de uma CCE de 208 visitas $x$ dia $^{-1}$ (Tabela 1).

Os fatores de correção relacionados aos aspectos climáticos brilho solar $\left(\mathrm{F}_{\mathrm{BS}}=(86,36 \%)\right.$ e precipitação $\left(F_{p}=74,20 \%\right)$ e foram os quesitos mais restritivos para o número de visitas (Tabela 1). Conforme Gois et al. (2019) o balanço hídrico dessa região apresenta distribuição irregular na chuva mensal, com estação chuvosa iniciando em setembro, com o maior excedente hídrico em janeiro e dezembro, períodos em que o fator de correção poderia ser mais limitante. Dacanal et al. (2010) quando compararam a percepção sobre conforto térmico em ambientes urbanos abertos e florestados no interior de São Paulo constataram que $75 \%$ dos entrevistados acharam os ambientes florestais mais frescos, considerando as variáveis microclimáticas. Para o público entrevistado, no geral, os ambientes florestados são percebidos como locais confortáveis, devido à "presença da natureza". A Região do médio Paraíba do Sul apresenta um clima com inverno seco e verão quente, diante disso as experiências em ambientes florestais podem representar uma alternativa de lazer bastante apropriada nos dias mais propícios ao longo do ano. Por outro lado, os fatores de correção que menos restringiram a visitação foram a capacidade de manejo $\left(F_{\mathrm{CM}}=41 \%\right)$ e a Acessibilidade $\left(F_{\mathrm{E}}=52,51 \%\right)$. Zanzini \& Macedo (1999) apud Bonatti et al. (2006) recomendam para áreas protegidas em países em desenvolvimento a adoção de um fator de capacidade de manejo de $30 \%$. A trilha possui uma declividade muito baixa (máxima $5^{\mathrm{O}}$ ), todavia quando associada à observação visual da compactação do solo seus efeitos podem ser ainda mais restritivos. Conforme Eisenlohr et al. (2013), a compactação do solo não é considerada na maioria dos métodos de cálculo de capacidade de carga e pode contribuir significativamente para acelerar os processos de erosão do solo, especialmente, nas áreas de maior declividade ou escassa cobertura vegetal. A sede da ARIE fica instalada a cerca de $3 \mathrm{~km}$ da unidade, o que demanda disponibilidade de transporte do órgão gestor para garantir o deslocamento dos usuários até a trilha. Nas duas cidades falta uma estratégia de marketing adequada para divulgação dos objetivos de conservação da Floresta da Cicuta para o público em geral. Dessa forma, o fortalecimento da visitação na ARIE da Floresta da Cicuta ainda precisa de muitos investimentos no sentido de tornar essa atividade acessível para a população, principalmente, de Volta Redonda e Barra Mansa. De acordo com Cimnaghia \& Mussini (2015), esses fatores atestam a baixa capacidade de manejo para o desenvolvimento do uso público na ARIE.

Tabela 1. Capacidade de carga turística para a visitação na Trilha Principal da ARIE da Cicuta, Volta Redonda, Barra Mansa, RJ.

\begin{tabular}{|c|c|c|}
\hline CCF (Eq. 1) & $\left(1 \times 1 \mathrm{~m}^{-2}\right) \times 1.753 \mathrm{~m}^{2} \times 2$ visitas $\times$ dia $^{-1} \times$ visitante $^{-1}$ & 3.506 visitas $x$ dia $^{-1}$ \\
\hline CCR (Eq. 2) & $3.506 \times 0,8636 \times 0,7376 \times 0,5251 \times 0,7421 \times 0,5820$ & 507 visitas $\times$ dia $^{-1}$ \\
\hline $\mathrm{FC}_{\mathrm{BS}}$ (Eq. 2.1) & $1-\left(3.656,96 \mathrm{~h} \times \mathrm{dia}^{-1} / 4.386,96 \mathrm{~h} \times \mathrm{dia}^{-1}\right)$ & 0,8636 \\
\hline $\mathrm{FC}_{\mathrm{E}}$ (Eq. 2.2) & $1-(140 m \times 2)+(60 m \times 3) / 1753 m)$ & 0,7376 \\
\hline $\mathrm{FC}_{\mathrm{A}}$ (Eq. 2.3) & $1-(832,5 \mathrm{~m} / 1753 \mathrm{~m})$ & 0,5251 \\
\hline $\mathrm{FC}_{\mathrm{P}}$ (Eq. 2.4) & 1 - (66,8 dias / 259 dias $)$ & 0,7420 \\
\hline $\mathrm{FC}_{\mathrm{F}}$ (Eq. 2.5) & 1 - (153 dias / 366 dias $)$ & 0,5820 \\
\hline $\mathrm{FC}_{\mathrm{ST}}(\mathrm{Eq} .2 .6)$ & $1-(0$ dias / 142 dias $) \times 100$ & - \\
\hline CCE & CCR $\times 41 \%$ & 208 visitas $\times$ dia $^{-1}$ \\
\hline
\end{tabular}


Conforme Limberger e Pires (2014), a metodologia de Cifuentes (1992) para estimar a capacidade de carga turística é amplamente utilizada no Brasil. Sendo associada à, aproximadamente, $60 \%$ das publicações, considerando periódicos e anais de congresso, durante o período de 1997 a 2014, a partir da avaliação de estudos relacionados ao manejo de impacto da visitação em áreas protegidas, segundo esses autores. A avaliação empírica prática pela equipe de gestão da ARIE compreende o limite de 40 visitantes $x$ dia $^{-1}$, ou seja, número inferior à capacidade de carga diária estimada por esse estudo (208 visitantes $\times \mathrm{dia}^{-1}$ ). Os estudos realizados para estimar a Capacidade de Carga em trilhas, utilizando a metodologia proposta por Cifuentes (1992) e suas adaptações, admitem resultados muito dispares, quando comparados entre eles, isso ocorre devido às diferenças ambientais observadas em de cada sítio (clima, fauna, vegetação); as características e capacidades de manejo (número de trilhas, tamanho do percurso, capacidade da equipe técnica, opções de atrativos/ intensidade de visitas) adotadas, além de fatores intrínsecos de cada trilha como: tipo de solo, relevo, cobertura do dossel e outras. Por exemplo, Sayan \& Atik (2011) encontraram um valor de 79 visitantes $\mathrm{x}$ dia $^{-1}$ para as trilhas do Parque Nacional de Termessos (Turquia), local com clima mediterrâneo e a maioria dos traçados com declividade superior a $20 \%$. Já Bonnatti et al. (2006) estimaram um valor de 19 visitantes $x$ dia $^{-1}$ na Floresta Nacional de São Francisco de Paula. (Rio Grande do Sul), localizada em ecossistema da Floresta Ombrófila, com topografia variada e clima chuvoso. Queiroz et al. (2014) ao avaliarem a capacidade de carga de duas trilhas em Açores (Portugal) registrando números bem diferentes em ambientes muito semelhantes, 118 visitantes $x$ dia $^{-1}$ o percurso para Lagoa do Fogo-Monte Escuro e 118 visitantes $\times$ dia $^{-1}$ para a trilha Ponta Delgada-Fajã Grande.

Cifuentes (1992) salienta que os valores obtidos para a Capacidade de Carga são relativos e dinâmicos, porque são circunstanciais. Limberger \& Pires (2014) ressalta que a Capacidade de Carga Turística (CCT) pode induzir os administradores das UCs a focarem o planejamento da visitação baseada, exclusivamente, em um número de visitas que os sítios atrativos possam receber com uma expectativa de baixo impacto, deixando de tomar providências administrativas e de manejo fundamentais para melhores práticas das atividades de educação, intepretação, turismo, lazer ou, mesmo, meramente contemplativas nesses espaços. A implementação de uma estratégia de gerenciamento que identifique especificamente essas condições e estabeleça padrões explícitos de qualidade será mais eficaz do que depender de capacidades de carga numérica. De acordo com McCool \& Lime (2001), é necessária a adoção de um método para avaliar capacidade de carga não apenas de forma numérica, mas uma estratégia de gerenciamento que identifique as condições e estabeleça padrões explícitos de qualidade será mais eficaz do que depender de capacidades de carga numérica.

Nesse sentido, Takahashi \& Cegana (2005); Kim et al. (2014); Limberger \& Pires (2014) descrevem modelos que se baseiam em processos de planejamento do uso público direcionados para alcançar os objetivos específicos dos sítios de visitação nas áreas protegidas. O espectro de oportunidades recreativas (ROS) segue um processo de planejamento flexível com seis etapas, e o ponto forte do ROS é o de garantir uma flexibilidade de oportunidades para a recreação, assim a ideia de diversificação na oferta leva à mitigação dos impactos nas áreas naturais, uma vez que as atividades possam ser direcionadas para os ambientes que as comportem. O limite aceitável de alteração (LAC) refere-se a um modelo para tomada de decisão que destaca a importância do manejo dos visitantes para alcançar os objetivos específicos das áreas de uso público das UCs, buscando identificar quais mudanças são aceitáveis nos ecossistemas. O processo de gestão da visitação (VAMP) objetiva integrar as necessidades dos visitantes com as oportunidades ofertadas por uma determinada área, através de uma abordagem do marketing.

McCool \& Lime (2001) ressaltam que esses métodos não são quantitativos e, também, não possuem rigor analítico, por isso sugerem a adoção de técnicas de modelagem não-linear e de dinâmica do Sistema, conciliando o entendimento sobre o fluxo com as principais modificações ambientais, com impacto sobre uso de energia, geração de resíduos sólidos, qualidade da água, manejo florestal, satisfação dos turistas, dentre outras variáveis que possam compor o modelo desses métodos.

Bennett (2016); Rodella et al. (2017) destacam a importância da avaliação da percepção dos visitantes diante de suas experiências em ambientes naturais, considerando distintas categorias, como impactos sociais da conservação, resultados ecológicos de conservação, legitimidade da governança de conservação e aceitabilidade do manejo de conservação.

Cisneros et al. (2016) recomendam o uso de Sistemas de Informações Geográficas (SIGs) para integrar as variáveis de interesses em bases espaciais para contribuir no processo de tomada de decisão na avaliação da Capacidade de Cargas em áreas de uso público.

Todavia, Medeiros \& Pereira (2011) afirmam que os Planos de Manejo em Unidades de Conservação baseiam-se no conceito de planejamento contínuo, gradativo, participativo e flexível. Conforme o SNUC, os Planos de Manejo de UCs precisam ser atualizados a cada cinco anos, no caso da ARIE da floresta da Cicuta em 2021. Sendo assim, a Metodologia de Cifuentes aplicado na Trilha Principal da ARIE não deve ser vista como uma panaceia, mas como um indicativo para ajudar na aplicação de métodos baseados nas experiências dos visitantes como, por exemplo, o LAC durante o processo de reavaliação do Plano de Manejo.

\section{Agradecimentos}

Os autores agradecem ao ICMBio pela autorização, ao acesso as informações e apoio logístico durante a realização deste trabalho, bem como, ao PPG Tecnologia Ambiental (UFF). 


\section{CONCLUSÃO}

O presente estudo demonstrou que a ARIE da Floresta da Cicuta está, relativamente, bem alinhada com as normativas gerais tratadas pelo SNUC;

A CCE encontrada para a trilha principal da ARIE (208 visitas $\mathrm{x} \mathrm{dia}{ }^{-1}$ ) sugere um bom manejo do uso público, considerado que o número empírico adotado pela equipe da UC não ultrapassa a 40 visitas $x$ dia $^{-1}$;

Esse estudo poderá contribuir com o processo de atualização do Plano de Manejo da ARIE, sendo complementado com outros estudos, por exemplo, o LAC.

\section{REFERÊNCIAS}

Amador, E., L. Cayot, M. Cifuentes, E. Cruz, F. Cruz \& P. Ayora. 1996. Determinación de la capacidad de carga turística en los sitios de visita del Parque Nacional Galápagos. Servicio Parque Nacional Galápagos, Ecuador. 42p. http://files.admonturistica.webnode.com.co/20000006781c3882bf6/Capacidad\%20de\%20carga.pdf

Bennett, N. J. 2016. Using perceptions as evidence to improve conservation and environmental management. Conservation Biology, 30: 582-592. https://onlinelibrary.wiley.com/doi/full/10.1111/cobi.1268 1

Bernard, E., L. A. Penna \& E. Araújo. 2014 Downgrading, downsizing, degazettement, and reclassification of protected areas in Brazil. Conservation Biology, 28: 939-950. https://onlinelibrary.wiley.com/doi/abs/10.1111/cobi.122 98

Bonatti, J., M. Marczwski, G. S. Rebelato, C. F. Silveira, F. D. Campello, G. G. Rodrigues \& S. M. Hartz. 2006. Trilhas da Floresta Nacional de São Francisco de Paula, Rio Grande do Sul, Brasil: mapeamento, análise e estudo da capacidade de carga turística. Revista Brasileira de Biociências, 4: 1-2. http://www.ufrgs.br/seerbio/ojs/index.php/rbb/article/vie w/20

BRASIL. Lei $N^{\circ}$ 9.985, de 18 Julho de 2000. Dispõe sobre Sistema Nacional de Unidades de Conservação da Natureza (SNUC). Publicado no Diário Oficial da União de 18 julho de 2000. http://www.mma.gov.br/port/conama/legiabre.cfm?codle gi $=322$

Canto-Silva, C. R. \& J. S. da Silva. 2017. Panorama da visitação e da condução de visitantes em Parques brasileiros. Revista Brasileira de Pesquisa em Turismo, 11: 365-386. https://rbtur.org.br/rbtur/article/view/1286

Cifuentes Arias, M. 1992. Determinación de capacidad de carga turística en áreas protegidas (No. 333.95 C569d). Turrialba, CR: CATIE.

Cimnaghi, E. \& P. Mussini. 2015. An application of tourism carrying capacity assessment at two Italian cultural heritage sites. Journal of Heritage Tourism, 10: 302-313.

https://www.tandfonline.com/doi/abs/10.1080/1743873X .2014 .988158

Cisneros, M. A. H., N. V. R. Sarmiento, C. A. Delrieux, M. C. Piccolo \& G. M. Perillo. 2016. Beach carrying capacity assessment through image processing tools for coastal management. Ocean \& Coastal Management, 130: 138-147. https://www.sciencedirect.com/science/article/pii/S0964 569116301193

Cupul-Magaña, A. L. \& A. P. Rodríguez-Troncoso. 2017. Tourist carrying capacity at Islas Marietas National Park: An essential tool to protect the coral community. Applied Geography, 88: 15-23. https://www.sciencedirect.com/science/article/pii/S0143 622817309256

Da Soller, J. \& C. Borghetti. 2013. Capacidade de Carga Turística: Um Estudo no Caminhos Rurais de Porto Alegre, RS. Rosa dos Ventos, 5: 511-527. http://www.redalyc.org/html/4735/473547094012/

Dacanal, C., L. C. Labaki \& T. M. L. D. Silva. 2010. Vamos passear na floresta! O conforto térmico em fragmentos florestais urbanos. Ambiente Construído. http://repositorio.unicamp.br/handle/REPOSIP/35347 Acesso em: 17 julho 2018.

Eisenlohr, P. V., L. Meyer, P. L. S. D. Miranda, V. L. Rezende, C. D. Sarmento, T. J. R. D. C. Mota, L.C. Garcia \& M. M. D. R. F. Melo. 2013. Trails and their ecological role: what have we learned and what are the prospects for restoration of ecosystems?. Hoehnea, 40: 407- 418. http://www.scielo.br/scielo.php?pid=S2236$89062013000300002 \&$ script $=$ sci_arttext

Fonseca, S. M. 2018. Influências de Unidades de Conservação na qualidade da água de corpos hídricos: Estudo de caso na ARIE Floresta da Cicuta, RJ. Dissertação (Mestrado em Tecnologia Ambiental) Universidade Federal Fluminense, Volta Redonda, RJ. Brasil.

http://www.pgta.uff.br/images/stories/dissertacoes/pgta \%20033.silvana\%20mendonca\%20da\%20fonseca.pdf Acessado em: 17 de jul 2018.

Gil, V. N., V. Gil \& A. M. Campo. 2014. Capacidad de carga turistica en el sendero del cerro ventana: Parque Provincial Ernesto Tornquist, Argentina. Estudios y perspectivas en turismo, 23: 362-375. http://www.scielo.org.ar/scielo.php?pid=S1851$17322014000200008 \&$ script $=$ sci_arttext\&tlng=pt

Gois, G., W. K. Freitas, P. M. B. Terassi, J. F. Oliveira-Júnior, A. Portz. 2019. variabilidade anual e mensal da chuva e da temperatura do ar no município de Resende, Rio de Janeiro. Revista Brasileira de Climatologia 15: 67-88. http://dx.doi.org/10.5380/abclima.v24i0.61611.

Ibañez Pérez, R. 2016. Capacidad de carga turística como base para el manejo sustentable de actividades ecoturísticas en Unidades de Manejo Ambiental (UMA) de Baja California Sur (BCS)". El periplo sustentable, 30:

http://www.scielo.org.mx/scielo.php?pid=S1870-

90362016000100037\&script=sci_arttext

Instituto Chico Mendes de Conservação da Biodiversidade - ICMBio. 2011. Avaliação comparada das aplicações do método RAPPAM nas Unidades de Conservação Federais, nos ciclos 2005-06 e 2010. Instituto Chico Mendes de Conservação da Biodiversidade, WWF-Brasil. Brasília: ICMBio, 134 p. Instituto Chico Mendes de Conservação da Biodiversidade - ICMBio. 2016. Plano de Manejo: ARIE Floresta da Cicuta. Volta Redonda: MMA/CSN, 118 p., 
Instituto Chico Mendes de Conservação da Biodiversidade - ICMBio. 2018. Painel Dinâmico de Informações sobre Unidades de Conservação Federais. MMA. Disponível em<http://qv.icmbio.gov.br/QvAJAXZ fc/opendoc2.htm?document=painel_corporativo_6476.q vw\&host=Local\&anonymous=true $>$. Acesso em: 17 jul. 2018.

Kim, S. O., B. Shelby \& M. D. Needham. 2014. Effects of facil ity developments and encounter levels on perceptions of settings, crowding, and norms in a Korean park. Environmental management, 53: 441-453. https://link.springer.com/article/10.1007/s00267-0130207-5

Limberger, P. F. \& P. dos Santos Pires. 2014. A aplicação das metodologias de capacidade de carga turística e dos modelos de gestão da visitação no Brasil. Revista de Turismo Contemporâneo, 2. ). https://periodicos.ufrn.br/turismocontemporaneo/article/ view/5473

Malik, M. I. \& M. S. Bhat. 2015. Sustainability of tourism development in Kashmir-ls paradise lost?. Tourism management perspectives, 16: 11-21. https://www.sciencedirect.com/science/article/abs/pii/S2 211973615000483

Marion, J. L., Y. F. Leung, H. Eagleston \& K. Burroughs. 2016. A review and synthesis of recreation ecology research findings on visitor impacts to wilderness and protected natural areas. Journal of forestry, 114:

352-362.

https://academic.oup.com/jof/article-

abstract/114/3/352/4599821

McCool, S. F. \& D. W. Lime. 2001. Tourism carrying capacity: tempting fantasy or useful reality?. Journal of sustainable tourism, 9: 372-388. https://www.tandfonline.com/doi/abs/10.1080/09669580 108667409

Medeiros, R. \& S. G. Pereira. 2011. Evolução e implementação dos planos de manejo em parques nacionais no estado do Rio de Janeiro. Revista Árvore, 35: 279-288.

http://www.scielo.br/pdf/rarv/v35n2/a12v35n2

MINISTÉRIO DO MEIO AMBIENTE - MMA. Diretrizes para Visitação em Unidades de Conservação. Brasília: Ministério do Meio Ambiente, 2006. 61 p. http://www.mma.gov.br/estruturas/ascom_boletins/_arq uivos/livro.pdf

Mitraud, S. Brasil, W. W. F. 2003. Manual de Ecoturismo de Base Comunitária-ferramentas para um planejamento responsável. 470p. WWF-World Wildlife Fund Brasil, Brasília, DF, Brasil. ISBN, 85-86440.

Nascimento, C. A., C. R. Canto-Silva, I. B. N. de Melo \& S. C. M. Marques. 2016. A regulamentação da atividade de condução de visitantes nos Sistemas Estaduais de Unidades de Conservação do Brasil. Revista Brasileira de Pesquisa em Turismo, 10: 516-532. https://www.rbtur.org/rbtur/article/view/1133

Queiroz, R. E., M. A. Ventura, J. A. Guerreiro \& R. T. D. Cunha. 2014. Capacidade de carga de trilhos pedestres inseridos em sítios da rede Natura 2000: um caso de estudo em ilhas do Atlântico Norte (Açores,
Portugal). Revista de Gestão Costeira Integrada, 14: 233-242. www.scielo.mec.pt/scielo.php?pid=S1646$88722014000200007 \&$ script=sci_arttext\&tlng=en

Ríos-Jara, E., C. M. Galván-Villa, F. A. RodríguezZaragoza, E. López-Uriarte \& V. T. MuñozFernández. 2013. The tourism carrying capacity of underwater trails in Isabel Island National Park, Mexico. Environmental management, 52: 335-347. https://link.springer.com/article/10.1007/s00267-013-

0047-3

Rodella, I., C. Corbau, U. Simeoni \& K. Utizi. 2017. Assessment of the relationship between geomorphological evolution, carrying capacity and users' perception: Case studies in Emilia-Romagna (Italy). Tourism Management, 59: 7-22. https://www.sciencedirect.com/science/article/pii/S0261 517716301170

Ruschmann, D. M., V. Doris, L. Paolucci \& N. A. Maciel. 2008. Capacidade de carga no planejamento turístico: Estudo de caso da Praia Brava-Itajaí frente à implantação do Complexo Turístico Habitacional Canto da Brava. Revista Brasileira de Pesquisa em Turismo, 2: 41-63. http://www.redalyc.org/html/5041/504152238004/

Sayan, M. S. \& M. Atik. 2011. Recreation carrying capacity estimates for protected areas: a study of Termessos National Park. Ekoloji, 20: 66-74. https://goo.gl/q31P5r

Soria Díaz, H. F. 2013. Determinación de la capacidad de carga turística en los sitios de visita de la Reserva Nacional Allpahuayo-Mishana, Loreto-Perú. 89pp. http://repositorio.unapiquitos.edu.pe/handle/UNAP/2450 Souza, G.R, A.L. Peixoto, M.J.B. Faria \& A.S. Zaú. 2007. Composição florística e aspectos estruturais do estrato arbustivo-arbóreo de um trecho de Floresta Atlântica no médio vale do Rio Paraíba do Sul, Rio de Janeiro, Brasil Sitientibus Série Ciências Biológicas, 7: 398-409.

http://www2.uefs.br/revistabiologia/pg7_n4.html

Souza, P. C. D. \& H. Lesjak Martos. 2008. Estudo do uso público e análise ambiental das trilhas em uma unidade de conservação de uso sustentável: Floresta Nacional de Ipanema, Iperó-SP. Revista Árvore, 32: 91100. http://www.redalyc.org/html/488/48813376011/

Takahashi, L. Y. \& A. C. Cegana. 2005. Como monitorar o impacto dos visitantes utilizando o sistema LAC-Limite Aceitável de Câmbio. Revista Turismo em Análise, 16:

https://www.revistas.usp.br/rta/article/view/63737

Watson, J. E., N. Dudley, D. B. Segan \& M. Hockings. 2014. The performance and potential of protected areas. Nature, 515: 67-73.

https://www.nature.com/articles/nature13947

Zanzini, A.C. \& R.L.G. Macedo. 1999. Determinação da capacidade de carga turística em uma trilha de interpretação, no Parque Florestal Quedas do Rio Bonito, Lavras, MG. IN: 3 o Simpósio Nacional de Ciências Florestais e Engenharia Ambiental, 1999, São Carlos: Escola de Engenharia de São Carlos. p. 203205. 